\title{
Indeterminism and Undecidability
}

\author{
Klaas Landsman \\ Department of Mathematics, Institute for Mathematics, Astrophysics, and \\ Particle Physics (IMAPP), Radboud University, Nijmegen, The Netherlands \\ Email: landsman@math.ru.nl
}

Dedicated to the memory of Michael Redhead (1929-2020)

\begin{abstract}
The aim of this paper is to argue that the (alleged) indeterminism of quantum mechanics, claimed by adherents of the Copenhagen interpretation since Born (1926), can be proved from Chaitin's follow-up to Gödel's (first) incompleteness theorem. In comparison, Bell's (1964) theorem as well as the so-called free will theorem-originally due to Heywood and Redhead (1983)-left two loopholes for deterministic hidden variable theories, namely giving up either locality (more precisely: local contextuality, as in Bohmian mechanics) or free choice (i.e. uncorrelated measurement settings, as in 't Hooft's cellular automaton interpretation of quantum mechanics). The main point is that Bell and others did not exploit the full empirical content of quantum mechanics, which consists of long series of outcomes of repeated measurements (idealized as infinite binary sequences): their arguments only used the long-run relative frequencies derived from such series, and hence merely asked hidden variable theories to reproduce single-case Born probabilities defined by certain entangled bipartite states. If we idealize binary outcome strings of a fair quantum coin flip as infinite sequences, quantum mechanics predicts that these typically (i.e. almost surely) have a property called 1-randomness in logic, which is much stronger than uncomputability. This is the key to my claim, which is admittedly based on a stronger (yet compelling) notion of determinism than what is common in the literature on hidden variable theories.
\end{abstract}

\section{Contents}

1 Introduction: Gödel and Bell 2

2 Randomness and its unprovability 4

3 Rethinking Bell's theorem 6

4 Are deterministic hidden variable theories deterministic? 8

5 Conclusion and discussion $\quad 10$

A The Born rule

B 1-Randomness

$\begin{array}{ll}\text { C Bell's theorem and free will theorem } & 18\end{array}$

References 21 


\section{Introduction: Gödel and Bell}

While prima facie totally unrelated, Gödel's theorem (1931) in mathematical logic and Bell's theorem (1964) in physics share a number of fairly unusual features (for theorems): 1

- Despite their very considerable technical and conceptual difficulty, both results are extremely famous and have caught the popular imagination like few others in science.

- Though welcome in principle-in their teens, many people including the author were intrigued by books with titles like Gödel, Escher Bach: An Eternal Golden Braid and The Dancing Wu-Li Masters: An Overview of the New Physics, both of which appeared in 1979-this imagination has fostered wild claims to the effect that Gödel proved that the mind cannot be a computer or even that God exists, whilst Bell allegedly showed that reality does not exist. Both theorems (apparently through rather different means) supposedly also supported the validity of Zen Buddhism2

- However, even among professional mathematicians (logicians excepted) few would be able to correctly state the content of Gödel's theorem when asked on the spot, let alone provide a correct proof, and similarly for Bell's theorem among physicists.

- Nonetheless, many professionals will be aware of the general feeling that Gödel in some sense shattered the great mathematician Hilbert's dream of what the foundations of mathematics should look like, whilst there is similar consensus that Bell dealt a lethal blow to Einstein's physical world view-though ironically, Gödel worked in the spirit and formalism of Hilbert's proof theory, much as Bell largely agreed with Einstein's views about quantum mechanics and about physics in general.

- Both experts and amateurs seem to agree that Gödel's theorem and Bell's theorem penetrate the very core of the respective disciplines of mathematics and physics.

In this light, anyone interested in both of these disciplines will want to know what these results have to do with each other, especially since mathematics underwrites physics (or at least is its language) 3 At first sight this connection looks remote. Roughly speaking 4

1. Gödel proved that any consistent mathematical theory (formalized as an axiomaticdeductive system in which proofs could in principle be carried out mechanically by a computer) that contains enough arithmetic is incomplete (in that arithmetic sentences $\varphi$ exist for which neither $\varphi$ nor its negation can be proved).

2. Bell showed that if a deterministic "hidden variable" theory underneath (and compatible with) quantum mechanics exists, then this theory cannot be local (in the sense that the hidden state, if known, could be used for superluminal signaling).

\footnotetext{
${ }^{1}$ In fact there are two incompleteness theorems in logic due to Gödel (see footnotes 2 and 5) and two theorems on quantum mechanics due to Bell (Brown \& Timpson, 2014; Wiseman, 2014), but for reasons to follow in this essay I am mainly interested in the first ones, of both authors, except for a few side remarks.

${ }^{2}$ See Franzén (2005) for an excellent first introduction to Gödel's theorems, combined with a fair and detailed critique of its abuses, including overstatements by both amateurs and experts (a similar guide to the use and abuse of Bell's theorems remains to be written), and Smith (2013) for a possible second go.

${ }^{3}$ Yanofsky (2013) nicely discusses both theorems in the context of the limits of science and reason.

${ }^{4}$ Both reformulations are a bit anachronistic and purpose-made. See Gödel (1931) and Bell (1964)!
} 
Both were triggered by a specific historical context. Gödel (1931) reflected on the recently developed formalizations of mathematics, of which he specifically mentions the Principia Mathematica of Russell and Whitehead and the axioms for set theory proposed earlier by Zermelo, Fraenkel, and von Neumann. Though relegated to a footnote, the shadow of Hilbert's program, aimed to prove the consistency of mathematics (ultimately based on Cantor's set theory) using absolutely reliable, "finitist" means, clearly loomed large, too 5

Bell, on the other hand, tried to understand if the de Broglie-Bohm pilot wave theory, which was meant to be a deterministic theory of particle motion reproducing all predictions of quantum mechanics, necessarily had to be non-local: Bell's answer, then, was "yes." 6

In turn, the circumstances in which Gödel and Bell operated had a long pedigree in the quest for certainty in mathematics and for determinism in physics, respectively 7 The former had even been challenged at least three times 8 first, by the transition from Euclid's mathematics to Newton's; second, by the set-theoretic paradoxes discovered around 1900 by Russell and others (which ultimately resulted from attempts to make Newton's calculus rigorous by grounding it in analysis, and in turn founding analysis in the real numbers and hence in set theory), and third, by Brouwer's challenge to "classical" mathematics, which he tried to replace by "intuitionistic" mathematics (both Hilbert and Gödel were influenced by Brouwer, though contrecoeur: neither shared his overall philosophy of mathematics).

In physics (and more generally), what Hacking (1990, Chapter 2) calls the doctrine of necessity, which thus far-barring a few exceptions-had pervaded European thought, began to erode in the 19th century, culminating in the invention of quantum mechanics between 1900-1930 and notably in its probability interpretation as expressed by Born (1926):

Thus Schrödinger's quantum mechanics gives a very definite answer to the question of the outcome of a collision; however, this does not involve any causal relationship. One obtains no answer to the question "what is the state after the collision," but only to the question "how probable is a specific outcome of the collision". (...) This raises the entire problem of determinism. From the standpoint of our quantum mechanics, there is no quantity that could causally establish the outcome of a collision in each individual case; however, so far we are not aware of any experimental clue to the effect that there are internal properties of atoms that enforce some particular outcome. Should we hope to discover such properties that determine individual outcomes later (perhaps phases of the internal atomic motions)? (...) I myself tend to relinquish determinism in the atomic world. (Born, 1926, p. 866, translation by the present author)

In a letter to Born dated December 4, 1926, Einstein's famously replied that 'God does not play dice' ('Jedenfalls bin ich überzeugt, daß der nicht würfelt'). Within ten years Einstein saw a link with locality 9 and Bell (1964) and later papers followed up on this.

\footnotetext{
${ }^{5}$ Gödel's second incompleteness theorem shows that one example of $\varphi$ is the (coded) statement that the consistency of the theory can be proved within the theory. This is often taken to refute Hilbert's program, but even among experts it seems controversial if it really does so. For Hilbert's program and its role in Gödel's theorems see e.g. Zach (2001), Tait (2005), Sieg (2013), and Tapp (2013).

${ }^{6}$ Greenstein (2019) is a popular book on the history and interpretation of Bell's work. Scholarly analyses include Redhead (1989), Butterfield (1992), Werner \& Wolf (2001), and the papers cited in footnote 1

${ }^{7}$ Some vocal researchers calim that Bell and Einstein were primarily interested in locality and realism, determinism being a secondary (or no) issue, but the historical record is ambiguous; more generally, over 10,000 papers about Bell's theorems show that Bell can be interpreted in almost equally many ways. But this controversy is a moot point: whatever his own (or Einstein's) intentions, Bell's (1964) theorem puts constraints on possible deterministic underpinnings of quantum mechanics, and that is how I take it.

${ }^{8}$ For an overall survey of this theme see Kline (1980).

${ }^{9}$ This phase in the history of quantum mechanics is described by Mehra \& Rechenberg (2000).
} 


\section{Randomness and its unprovability}

This precise history has a major impact on my argument, since it shows that right from the beginning the kind of randomness that Born (probably preceded by Pauli and followed by Bohr, Heisenberg, Jordan, Dirac, von Neumann, and most of the other pioneers of quantum mechanics except Einstein, de Broglie, and Schrödinger) argued for as being produced by quantum mechanics, was antipodal to determinism 10 Thus randomness in quantum mechanics was identified with indeterminism, and hence attempts (like the de Broglie-Bohm pilot wave theory) to undermine the "Copenhagen" claim of randomness looked for deterministic (and arguably realistic) theories underneath quantum mechanics.

Although "undecidability" may sound a bit like "indeterminism", the analogy between the quests for certainty in mathematics and for determinism in physics (and their alleged undermining by Gödel's and Bell's theorems, respectively) may sound rather superficial. To find common ground more effort is needed to bringing these theorems together 11

First, some of its "romantic" aspects have to be removed from Gödel's theorem, notably its reliance on self-reference, although admittedly this was the key to both Gödel's original example of an undecidable sentence $\varphi$ (which in a cryptic way expresses its own unprovability) and his proof, in which an axiomatic theory that includes arithmetic is arithmetized through a numerical encoding scheme so as to be able to "talk about itself". Though later proofs of Gödel's theorem also use numerical encodings of mathematical expressions (such as symbols, sentences, proofs, and computer programs), this is done in order to make recursion theory (initially a theory of functions $f: \mathbb{N} \rightarrow \mathbb{N}$ ) available to a wider context, rather than to exploit self-reference. Each computably enumerable but uncomputable subset $E \subset \mathbb{N}$ leads to undecidable statements (very rarely in mainstream mathematics) 12 namely those for which the sentence $n \notin E$ is true but unprovable. Chaitin's (first) incompleteness theorem (Theorem B.1 in Appendix B), which will play an important role in my reasoning, is an example of this. To understand this theorem and its background we return to the history of 20th century mathematics and physics.

Hilbert influenced this history in many ways 13 of which the sixth problem on his famous list of 23 mathematical problems from 1900 is particularly relevant here: this problem concerns the "Mathematical Treatment of the Axioms of Physics, especially the theory of probabilities and mechanics' (Hilbert, 1902). This problem influenced our topic in two initially independent ways, which now come together. First, the problem inspired von Neumann (1932) to develop his mathematical axiomatization of quantum mechanics, which still forms the basis of all mathematically rigorous work on this theory. In particular, he initiated the literature on hidden variable theories (see \$3). Second, it led both von Mises and Kolmogorov to their ideas on the mathematical foundations of probability and randomness, initially in opposite ways: whereas von Mises (1919) tried (unsuccessfully) to first axiomatize random sequences of numbers and then extract probability from these as asymptotic relative frequency, Kolmogorov (1933) successfully axiomatized probability first and then (unsuccessfully) sought to extract some notion of randomness from this.

\footnotetext{
${ }^{10}$ See Landsman (2020) for the view that randomness is a family resemblance (in that it lacks a meaning common to all its applications) with the special feature that its various uses are always defined antipodally.

${ }^{11}$ Also cf. e.g. Breuer (2001), Calude (2004), Svozil, Calude \& Stay (2005), and Szangolies (2018).

${ }^{12}$ A subset $E \subset \mathbb{N}$ is computably enumerable (c.e.) if it is the image of a computable function $f: \mathbb{N} \rightarrow \mathbb{N}$, and computable if its characteristic function $1_{E}$ is computable, which is true iff both $E$ and $\mathbb{N} \backslash E$ are c.e.

${ }^{13}$ This is true for physics almost as much as it is (more famously) for mathematics, since Hilbert played a major role in the mathematization of the two great theories of twentieth century physics, i.e. general relativity (Corry, 2004; Renn, 2007) and quantum mechanics (Rédei \& Stöltzner, 2001; Landsman, 2021).
} 
The basic problem (already known to Laplace and perhaps even earlier probabilists) was that, in a 50-50 Bernoulli process for simplicity, an apparently "random" string like

$$
\sigma=001101010111010010100011010111
$$

is as probable as a "deterministic" string like

$$
\sigma=11111111111111111111111111111 .
$$

In other words, their probabilities say little or nothing about the "randomness" of individual outcomes. Imposing statistical properties helps but is not enough to guarantee randomness. It is slightly easier to explain this in base 10, to which I therefore switch for a moment. If we call a sequence $x$ Borel normal if each possible string $\sigma$ in $x$ has relative frequency $10^{-|\sigma|}$, where $|\sigma|$ is the length of $\sigma$ (so that each digit $0, \ldots, 9$ occurs $10 \%$ of the time, each block 00 to 99 occurs $1 \%$ of the time, etc.), then Champernowne's number

$$
0123456789101112131415161718192021222324252629282930 \ldots
$$

can be shown to be Borel normal. The decimal expansion of $\pi$ is conjectured to be Borel normal, too (and has been empirically verified to be so in billions of decimals), but these numbers are hardly random: they are computable, which is one version of "deterministic".

Any sound definition of randomness (for binary strings or sequences) has to navigate between Scylla and Charybdis: if the definition is too weak (such as Borel normality), counterexamples will undermine it (such as Champernowne's number), but if it is too strong (such as being lawless, like Brouwer's choice sequences), it will not hold almost surely in a 50-50 Bernoulli process (Moschovakis, 2016). As an example of such sound navigation, Solomonoff, Kolmogorov, Martin-Löf, Chaitin, Levin, Solovay, Schnorr, and others developed the algorithmic theory of randomness (Li \& Vitányi, 2008). The basic idea is that a string or sequence is random iff its shortest description is the sequence itself, but the notion of a description has to made precise to avoid Berry's paradox:

The Berry number is the smallest positive integer that cannot be described in less than eighteen words.

The paradox, then, is that on the one hand this number must exist, since only finitely many integers can be described in less than eighteen words and hence the set of such numbers must have a lower bound, while on the other hand Berry's number cannot exists by its own definition 14 In the case at hand, the notion of a description is sharpened by asking it to be computable, so that, roughly speaking (see appendix B for technical details), we call a (finite) binary string $\sigma$ (Kolomogorov) random if the length of the shortest computer program generating $\sigma$ is at least as long as $\sigma$ itself, and call an (infinite) binary sequence $x$ (Levin-Chaitin) random or 1-random if its (sufficiently long) finite truncations are Kolomogorov random. At last, for finite strings $\sigma$ Chaitin's (first) incompleteness theorem states that although countably many strings $\sigma$ are random, this can be proved only for finitely many of these, whereas for infinite sequences $x$ his (second) incompleteness theorem says that if such a sequence is random, only finitely many of its digits can be computed (see Theorems B.1 andB.4 for precise statements). Thus randomness is elusive.

\footnotetext{
${ }^{14}$ This is one of innumerable paradoxes of natural language, which leads to an incompleteness theorem once the notion of a description has been appropriately formalized in mathematics, much as Gödel's first incompleteness theorem turns the the liar's paradox into a theorem.
} 


\section{Rethinking Bell's theorem}

In order to locate Bell's (1964) theorem in the literature on quantum mechanics and (in)determinism, I recall that Hilbert's sixth problem inspired both the work of von Mises and Kolmogorov that eventually gave rise to the algorithmic theory of randomness, and (Hilbert's postdoc) von Neumann's work on the mathematical foundations of quantum mechanics, culminating in his book (von Neumann, 1932). One of his results was that there can be no nonzero function $\lambda: H_{n}(\mathbb{C}) \rightarrow \mathbb{R}$ (where $H_{n}(\mathbb{C})$ is the space of hermitian $n \times n$ matrices, seen as the observables of a quantum-mechanical $n$-level system) that is:

1. dispersion-free (i.e. $\lambda\left(a^{2}\right)=\lambda(a)^{2}$ for each $\left.a \in H_{n}(\mathbb{C})\right)$;

2. linear (i.e. $\lambda(s a+t b)=s \lambda(a)+t \lambda(b)$ for all $s, t \in \mathbb{R}$ and $a, b \in H_{n}(\mathbb{C})$ ).

Unfortunately, von Neumann interpreted this correct, non-circular, and interesting result as a proof that quantum mechanics is complete in the sense that there can be no hidden variables in the sense of Born (1926), i.e. 'properties that determine individual outcomes'. The reason this does not follow is twofold 15 First, the proof relies on a tacit assumption that later came to be called non-contextuality, namely that the value $\lambda(a)$ of some observable $a$ only depends on $a$, whereas measurement ideology à la Bohr (1935) suggests that it may depend on a measurement context, formalized as a further set of observables commuting with $a$ (unless $a$ is maximal such a set is far from unique) 16 Second, though natural, the linearity assumption is very strong and excludes even eigenvalues of $a$.

This second point was remedied by the Kochen-Specker theorem 17 who weakened von Neumann's linearity assumption to linearity on commuting observables, which at least incorporates eigenvalues and is even found so appealing that the Kochen-Specker is generally taken to exclude non-contextual hidden variable theories. See also Appendix C.

The final step in the series of attempts, initiated by von Neumann, to exclude hidden variables by showing that subject to reasonable assumptions the corresponding value attributions cannot exist even independently of any statistical considerations, is the so-called free will theorem 18 In the wake of the renowned "EPR" paper (Einstein, Podolsky and Rosen, 1935) the setting has now become bipartite (i.e. Alice and Bob who are spacelike separated each perform experiments on a correlated state) and the non-contextuality assumption is weakened to local contextuality: the outcomes of Alice's measurements are independent of any choice of measurements Bob might perform, and vice versa 19 Thus her value attributions $\lambda(a \mid$ context $)$ may well be contextual, as long as the observables commuting with the one she measures (i.e. a), which form a context to a, are local to her.

A second line of research, which goes back at least to de Broglie (1928), was influentially taken up by Bohm (1952), and most recently includes 't Hooft (2016), assumes the possibility of non-contextual value attributions and tries to make these compatible with the Born rule of quantum mechanics. Bell (1964) was primarily concerned with such theories, asking himself if a deterministic theory like Bohm's was necessarily non-local.

\footnotetext{
${ }^{15}$ See also Bub (2011), Dieks (2016), and forthcoming work by Chris Mitsch for balanced accounts.

${ }^{16}$ The idea of contextuality was first formulated by Grete Hermann (Crull \& Bacciagaluppi, 2016).

${ }^{17}$ See Kochen \& Specker (1967). Ironically, his followers attribute this theorem to Bell (1966), although the result is just a technical sharpening of von Neumann's result they so vehemently ridicule. For a deep philosophical analysis of the Kochen-Specker theorem, as well as of Bell's theorems, see Redhead (1989).

${ }^{18}$ See appendix C. This theorem is originally due to Heywood \& Redhead (1983), with follow-ups by Stairs (1983), Brown \& Svetlichny (1990), and Clifton (1993), but it was named and made famous by Conway \& Kochen (2009), whose main contribution was an emphasis on free will (Landsman, 2017, Chapter 6).

${ }^{19}$ Since Alice and Bob are spacelike separated their observables commute (Einstein locality).
} 
In Bell's analysis, which takes place in the bipartite (EPR) setting, the quantum-mechanical probabilities are obtained by formally averaging over the set of hidden variables, i.e.,

$$
P_{\psi}(F=x, G=y \mid A=a, B=b)=\int_{\Lambda} d \mu_{\psi}(\lambda) P_{\lambda}(F=x, G=y \mid A=a, B=b) .
$$

Here $\psi$ is some (explicitly identified) quantum state of a correlated pair of (typically) 2-level quantum systems (which may be either optical, where the degree of freedom is helicity, or massive, where the degree of freedom is spin), $F$ is an observable measured by Alice defined by her choice of setting $a$, likewise $G$ for Bob defined by his setting $b$, with possible outcomes $x \in \underline{2}=\{0,1\}$, likewise $y \in \underline{2}$ for Bob; the left-hand side is the Born probability for the outcome $(x, y)$ if the correlated system has been prepared in the state $\psi$; the expression $P_{\lambda}(\cdots)$ on the right-hand side is the probability of the outcome $(x, y)$ if the unknown hidden variable or state equals $\lambda$, and finally, $\mu_{\psi}$ is some probability measure on the space $\Lambda$ of hidden states supposedly provided by the theory for each state $\psi$.

We now say that the hidden variable theory supplying the above quantities is:

- deterministic if the probabilities $P_{\lambda}(F=x, G=y \mid A=a, B=b)$ equal 0 or 1 ;

- locally contextual if the expression

$$
P_{\lambda}(F=x \mid A=a, B=b)=\sum_{y=0,1} P_{\lambda}(F=x, G=y \mid A=a, B=b) ;
$$

is independent of $b$, whilst the corresponding expression

$$
P_{\lambda}(G=y \mid A=a, B=b)=\sum_{x=0,1} P_{\lambda}(F=x, G=y \mid A=a, B=b),
$$

is independent of $a$. That is, the probabilities of Alice's outcomes are independent of Bob's settings, and vice versa. This locality property seems very reasonable and in fact it follows from special relativity, for if Bob chooses his settings just before his measurement, there is a frame of reference in which Alice measures before Bob has chosen his settings, and vice versa. In turn, this is equivalent to the property that even if she knew the value of $\lambda$, Alice could not signal to Bob, and vice versa 20

Bell proved that a hidden variable theory cannot satisfy (3.1) and be both deterministic and locally contextual (which explained why Bohm's theory had to be non-local). Making his tacit assumption that experimental settings can be "freely" chosen explicit, we obtain 21

Theorem 3.1 The conjunction of the following properties is inconsistent:

1. determinism;

2. quantum mechanics, i.e. the Born rule for $P_{\psi}(F=x, G=y \mid A=a, B=b)$;

3. local contextuality;

4. free choice, i.e. (statistical) independence of the measurement settings a and $b$ from each other and from the hidden variable $\lambda$ (given the probability measure $\mu_{\psi}$ ).

\footnotetext{
${ }^{20}$ In quantum mechanics the left-hand side of (3.1) satisfies this locality condition for any state $\psi$.

${ }^{21}$ See Landsman (2017), $\S 6.5$ for details, or Appendix C below for a summary.
} 


\section{Are deterministic hidden variable theories deterministic?}

Although the assumptions have a slightly different meaning, the free will theorem leads to the same result as Bell's theorem (see Appendix C), so that the (no) hidden variable tradition initiated by von Neumann, which culminates in the former, coalesces with the (positive) hidden variable tradition going back to de Broglie, shown its place by the latter. Thusly there are the obvious four (minimal) ways out of the contradiction in Theorem 3.1 .

- Copenhagen (i.e. mainstream) quantum mechanics rejects determinism;

- Valentini (2019) rejects the Born rule and hence QM (see the end of §5 below);

- Bohmians reject local contextuality 22

- 't Hooft (2016) rejects free choice.

We focus on the last two options, so that determinism and quantum mechanics (i.e. the Born rule) are kept. In both cases the Born rule is recovered by averaging the hidden variable with respect to a probability measure $\mu_{\psi}$ on the space of hidden variables, given some (pure) quantum state $\psi$. The difference is that in Bohmian mechanics the total state (which consists of the hidden configuration plus the "pilot wave" $\psi$ ) determines the measurement outcomes given the settings, whereas in 't Hooft's theory the hidden variable determines the outcomes as well as the settings 23 More specifically:

- In Bohmian mechanics the hidden variable is position $q$, and $d \mu_{\psi}(q)=|\psi(q)|^{2} d q$ is the Born probability for outcome $q$ with respect to the expansion $|\psi\rangle=\int d q \psi(q)|q\rangle$.

- In 't Hooft's theory the hidden variable is a basis vector $|m\rangle$ in some separable Hilbert space $H(m \in \mathbb{N})$, and once again the measure $\mu_{\psi}(m)=\left|c_{m}\right|^{2}$ is given by the Born probability for outcome $m$ with respect to the expansion $|\psi\rangle=\sum_{m} c_{m}|m\rangle$.

Thus the hidden variables (i.e. $q \in Q$ and $m \in \mathbb{N}$, respectively) have familiar quantummechanical interpretations and also their compatibility measures are precisely the Born measures for the quantum state $\psi$. In this light, we may ask to what extent these hidden variable theories are truly deterministic, as their adherents claim them to be. Since the argument does not rely on entanglement and hence on a bipartite experiment, we may as well work with a quantum coin toss. The settings, possible contexts, and quantum state of the experiments are then fixed, so that only the hidden state $\lambda$ may change and hence we may treat Bohmian mechanics and 't Hooft's theory on the same footing. Idealizing to an infinite run, one has an outcome sequence $x: \mathbb{N} \rightarrow \underline{2}$. Standard (Copenhagen) quantum mechanics refuses to say anything about its origin, but nonetheless it does make very specific predictions about $x$. The basis of these predictions is the following theorem, whose notation and proof are explained in Appendix A. One may think of a fair quantum coin, in which $\sigma(a)=\underline{2}=\{0,1\}$ and $\mu_{a}(0)=\mu_{a}(1)=1 / 2$, and which probabilistically is indistinguishable from a fair classical coin (which in my view cannot exist, cf. \$5).

\footnotetext{
${ }^{22}$ There is a subtle difference between Bohmian mechanics as reviewed by e.g. Goldstein (2017), and de Broglie's original pilot wave theory (Valentini, 2019). This difference is immaterial for my discussion.

${ }^{23}$ In Bohmian mechanics, the hidden state $q \in Q$ just pertains to the particles undergoing measurement, whilst the settings $a$ are supposed to be "freely chosen" for each measurement (and in particular are independent of $q$ ). The outcome is then fixed by $a$ and $q$. In 't Hooft's theory, the hidden state $x \in X$ of "the world" determines the settings as well as the outcomes. Beyond the issue raised in the main text, Bohmians (but not 't Hooft!) therefore have an additional problem, namely the origin of the settings (which are simply left out of the theory). This weakens their case for determinism even further.
} 
Theorem 4.1 The following procedures for repeated identical independent measurements are equivalent (in giving the same possible outcome sequences with the same probabilities):

1. Quantum mechanics is applied to the whole run, described as a single quantummechanical experiment with a single classically recorded outcome sequence;

2. Quantum mechanics is applied to single experiments (with classically recorded outcomes), upon which classical probability theory takes over to combine these.

Either way, the (purely theoretical) Born probability $\mu_{a}$ for single outcomes induces the infinite Bernoulli process probability $\mu_{a}^{\infty}$ on the space $\sigma(a)^{\mathbb{N}}$ of infinite outcome sequences.

Theorem B.3 in Appendix B then implies:

Corollary 4.2 With respect to the "fair" probability measure $P^{\infty}$ on $\underline{2}^{\mathbb{N}}$ almost every outcome sequence $x$ of an infinitely often repeated fair quantum coin flip is 1-random.

In hidden variable theories, on the other hand, $x$ factors through $\Lambda$, that is 24 there are functions $h: \mathbb{N} \rightarrow \Lambda$ and $g: \Lambda \rightarrow \underline{2}$ such that $x=g \circ h$. Hidden variable theories do provide $g$, i.e. describe the outcome of any experiment given the value of the hidden variable $\lambda \in \Lambda$. However, what about $h$, that is, the specification of the value of the hidden variable $\lambda$ in each run of the experiment? There are just the following two scenarios:

1. The function $h$ is provided by the hidden variable theory. In that case, since the theory is supposed to be deterministic, $h$ explicitly gives the values $\lambda_{n}=h(n)$ for each $n \in \mathbb{N}$ (i.e. experiment no. $n$ in the run). Since $g$ is also given, this means that $x$ is given by the theory. By Theorem B.4 (i.e. Chaitin's second incompleteness theorem), the outcome sequence cannot be 1-random, against Corollary 4.2 ,

2. The function $h$ is not provided by the hidden variable theory. In that case, the theory fails to determine the outcome of any specific experiment and just provides averages of outcomes. My conclusion would be that, except for some kind of a "story", nothing has been gained over quantum mechanics, but hidden variable theorists argue that their theories cannot be expected to provide initial conditions (for experiments), and claim that the randomness in measurement outcomes originates in the randomness of the initial conditions of the experiment 25 But then the question arises what else provides these conditions, and hence our function $h$. The point here is that in order to recover the predictions of quantum mechanics as meant in Corollary 4.2 , the function $h$ must sample the Born measure (in its guise of the compatibility measure $\mu_{\psi}$ on $\Lambda$ ), in the sense of "randomly" picking elements from $\Lambda$, distributed according to $\mu_{\psi}$, cf. (A.2). This, in turn, should guarantee that the sequences $x=g \circ h$ mimic fair coin flips. Since $g$ is supposed to be given, this implies that the randomness properties of $x$ must entirely originate in $h$. This origin cannot be deterministic, since in that case we are back to the contradictory scenario 1 above. Hence $h$ must come from some unknown external random process in nature that our hidden variable theories invoke as a kind of an oracle. In my view the need for such a random oracle undermines their purpose and makes them self-defeating. Every way you look at this you lose!

\footnotetext{
${ }^{24}$ The function $g$ incorporates all details of the experiment that may affect the outcome (like the setting, context, and quantum state) except the hidden variable $\lambda$ (which it specifies). It has nothing to do with non-contextual value assignments on the set of quantum-mechanical observables (which do not exist).

${ }^{25}$ The Bohmians are divided on the origin of their compatibility measure, referred to in this context as the quantum equilibrium distribution, cf. Dürr, Goldstein, \& Zanghi (1992) against Valentini (2019). The origin of $\mu_{\psi}$ is not my concern, which is the need to randomly sample it and the justification for doing so.
} 


\section{Conclusion and discussion}

We may summarize the discussion in the previous section as follows 26

Theorem 5.1 For any hidden variable theory $T$ the following properties are incompatible:

1. Determinism: $T$ states the outcome of the measurement of any observable a given the value $\lambda \in \Lambda$ of the hidden variable via a function $g: \Lambda \rightarrow \sigma(a)$ and provides these values for each experiment; for an infinite run this is done via some function $h: \mathbb{N} \rightarrow \Lambda$, so that $T$ provides the outcome sequence $x: \mathbb{N} \rightarrow \sigma(a)$ through $x=g \circ h$.

2. Born rule: Outcome sequences are almost surely 1-random. (cf. Corollary 4.2).

The proof is short. According to the first clause $T$ states the entire outcome sequence $x$. By Chaitin's incompleteness theorem B.4 this is incompatible with the second clause.

In order to understand Theorem 5.1 and its proof it may be helpful to note that in classical coin tossing the role of the hidden state is also played by the initial conditions (cf. Diaconis \& Skyrms, 2018 Chapter 1, Appendix 2). The 50-50 chances (allegedly) making the coin fair are obtained by averaging over the initial conditions, i.e., by sampling. By the same arguments, this sampling cannot be deterministic, i.e. given by a function like $h$, for otherwise the outcome sequences appropriate to a fair coin would not obtain: it must be done in a genuinely random way and hence by appeal to an external random process. This is impossible classically, so that-unless they have a quantum-mechanical seed-fair classical coins do not exist, as confirmed by Diaconis \& Skyrms (2018, Chapter 1).

I conclude that deterministic hidden variable theories compatible with quantum mechanics do not exist. The reason that Bell's (1964) theorem and the free will theorem leave two loopholes for determinism (i.e. local contextuality and no free choice) is that their compatibility condition with quantum mechanics is stated too weakly: the theory is only required to reproduce certain single-case (Born) probabilities, as opposed to the properties of typical outcome sequences (from which the said probabilities are extracted as long-run frequencies). This reason this approach is still partly successful lies in the clever use of entangled states. If one rejects the second requirement on determinism in Theorem 5.1, Bell's theorem and the free will theorem still provide useful constraints on deterministic hidden variable theories, but as shown in the previous section such a rejection necessitates an appeal to an unknown random process and hence seems self-defeating.

Let us now consider the role of the idealization to infinite outcome sequences and see what happens if the experimental runs are finite 27 Once again, via Theorem 4.1 the Born rule predicts that outcome strings will be Kolmogorov random with high probability. Any deterministic theory (in the sense of Theorem 5.1) provides an explicit description (say in ZFC) of the outcomes, whose randomness would be provable from this description. But this is precluded by Chaitin's first incompleteness theorem (i.e. Theorem B.1), now in the role played by his second incompleteness theorem in the infinite case 28

\footnotetext{
${ }^{26}$ In stating the second condition I have taken $\sigma(a)=\{0,1\}$ with 50-50 Born probabilities, but this can be generalized to other spectra and probability measures. See Downey \& Hirschfeldt (2010), §6.12.

${ }^{27}$ In other words, we examine whether Earman's principle is satisfied, cf. footnote 38 .

${ }^{28}$ To make this argument completely rigorous one would need to define what a "description" provided by a deterministic theory means logically. There is a logical characterization of deterministic theories (Montague, 1974), and there are some arguments to the effect that the evolution laws in deterministic theories should be computable, cf. Earman (1986), Chapter 11, and Pour-El \& Richards (2016), passim, but this literature makes no direct reference to output strings or sequences of the kind we analyze and in
} 
Nonetheless, although their incompatibility with quantum mechanics has now been established, it will be hard to disprove deterministic hidden variable theories from experimental data. Let us look at the proof of Bell's theorem for inspiration as to what such a (dis)proof should look like. In the context of the EPR-Bohm experiment local deterministic hidden variable theories predict correlations that satisfy the Bell inequalities 29 whereas on suitable settings quantum mechanics predicts (and experiment shows) that typical outcome sequences violate these inequalities. Now a disproof of some deterministic hidden variable theory $T$ cannot perhaps be expected to show that all quantum-mechanical outcome sequences violate the predictions of the hidden variable theory (indeed they do not, albeit with low probability), but it should identify at least a sufficiently large number of typical (i.e. random) sequences. However, even in the finite case this identification is impossible by Theorem B.1, so that the false predictions of $T$ cannot really be confronted with the correct predictions of quantum mechanics. Thus the unprovability of their falsehood condemns deterministic hidden variable theories, and perhaps even determinism as a whole, to a zombie-like existence in a twilight zone comparable with the Dutch situation around selling soft drugs: although this is forbidden by law, it is (officially) not prosecuted.

The situation would change drastically if deterministic hidden variable theories gave up their compatibility with the Born rule (on which my entire reasoning is based), as for example Valentini (2019) has argued in case of the de Broglie-Bohm pilot wave theory. For it is this compatibility requirement that kills such theories, which could leave zombie-dom if only they were brave enough to challenge the Born rule. This might open the door to superluminal signaling and worse, but on the other hand the possibility of violating the Born rule would also provide a new context for deriving it, e.g. as a dynamical equilibrium condition (as may be the case for the Broglie-Bohm theory, if Valentini is right).

I would personally expect that the Born rule is emergent from some lower-level theory, which equally well suggests that it is valid in some limit only, rather than absolutely.

The author is grateful to Jacob Barandes, Jeremy Butterfield, Cristian Calude, Erik Curiel, John Earman, Bas Terwijn, and Noson Yanofsky, as well as to members of seminar audiences and especially readers of the first version of this essay on the FQXi website for very helpful comments and corrections. He is even more grateful to the late Michael Redhead, for his exemplary approach to the foundations of physics.

any case the identification of "deterministic" with "computable" is obscure even in situations where the latter concept is well defined. For example, if we stipulate that $h: \mathbb{N} \rightarrow \Lambda$ is computable (and likewise $g: \Lambda \rightarrow 2$ ) then the above appeal to Chaitin's first incompleteness theorem is not even necessary, but this seems too easy. A somewhat circular solution, proposed by Scriven (1957), is to simply say that $T$ is deterministic iff the output strings or sequences it describes are not random, but this begs for a more explicit characterization. One might naively expect such a characterization to come from the arithmetical hierarchy (found in any book on computability): if, as before, we identify $\underline{2}^{\mathbb{N}}$ with the power set $P(\mathbb{N})$ of $\mathbb{N}$, then $S \subset \mathbb{N}$ is called arithmetical if there is a formula $\psi(x)$ in PA (Peano Arithmetic) such that $n \in S$ iff $\mathbb{N} \vDash \psi(n)$, that is, $\psi(n)$ is true in the usual sense. We may then classify the arithmetical subsets through the logical form of $\psi$, assumed in prenex normal form (i.e., all quantifiers have been moved to the left): $S$ is in $\Sigma_{0}^{0}=\Pi_{0}^{0}$ iff $\psi$ has no quantifiers or only bounded quantifiers (in which case $S$ is computable), and then recursively $S \in \Sigma_{n+1}^{0}$ iff $\psi(x)=\exists_{y} \varphi(x, y)$ with $\varphi \in \Pi_{n}^{0}$, and $\varphi \in \Pi_{n+1}^{0}$ iff $\psi(x)=\forall_{y} \varphi(x, y)$ with $\varphi \in \Sigma_{n}^{0}$. Here any singly quantified expression $\exists_{y} \varphi(x, y)$ may be replaced by $\exists y_{1} \cdots \exists y_{k} \varphi\left(x, y_{1}, \ldots, y_{k}\right)$ and likewise for $\forall_{y}$. By convention $\Sigma_{n}^{0} \subset \Sigma_{n+1}^{0}$ and $\Pi_{n}^{0} \subset \Pi_{n+1}^{0}$, and $\Delta_{n}^{0}:=\Sigma_{n}^{0} \cap \Pi_{n}^{0}$. Since in classical $\operatorname{logic} \forall_{y} \varphi(x, y)$ is equivalent to $\neg \exists_{y} \neg \varphi(x, y)$, it follows that $\Pi_{n}^{0}$ sets are the complements of $\Sigma_{n}^{0}$ sets. One would then like to locate deterministic theories somewhere in this hierarchy, preferably above the computable $\Delta_{0}^{0}$. The idea of a hidden variable (namely $y$ ) suggests $\Sigma_{1}^{0}$ and closure under complementation (it would be crazy if some deterministic theory prefers ones over zeros) then leads to $\Delta_{1}^{0}$, but this equals $\Delta_{0}^{0}$. The next level $\Delta_{2}^{0}$ is impossible since this already contains 1-random sets like Chaitin's $\Omega$. Hence more research is needed.

${ }^{29}$ For Bell's proof it is irrelevant whether or not some hidden variable is able to sample the compatibility measure, since the Bell inequalities follow from pointwise bounds, cf. Landsman (2017), eq. (6.119). 


\section{A The Born rule}

The Born measure is a probability measure $\mu_{a}$ on the spectrum $\sigma(a)$ of a (bounded) selfadjoint operator $a$ on some Hilbert space $H$, defined as follows by any state $\omega$ on $B(H) .30$

Theorem A.1 Let $H$ be a Hilbert space, let $a^{*}=a \in B(H)$, and let $\omega$ be a state on $B(H)$. There exists a unique probability measure $\mu_{a}$ on the spectrum $\sigma(a)$ of a such that

$$
\omega(f(a))=\int_{\sigma(a)} d \mu_{a}(\lambda) f(\lambda), \text { for all } f \in C(\sigma(a)) .
$$

The Born measure is a mathematical construction; what is its relationship to experiment? This relationship must be the source of the (alleged) randomness of quantum mechanics, for the Schrödinger equation is deterministic. We start by postulating, as usual, that $\mu_{a}(\Delta)$ is the (single case) "probability" that measurement of the observable $a$ in the state $\omega$ (which jointly give rise to the pertinent Born measure $\mu_{a}$ ) gives a result $\lambda \in \Delta \subset \sigma(a)$. Here I identify single-case "probabilities" with numbers (consistent with the probability calculus) provided by theory, upon which long-run frequencies provide empirical evidence for the theory in question, but do not define probabilities. The Born measure is a case in point: these probabilities are theoretically given, but have to be empirically verified by long runs of independent experiments. In other words, by the results reviewed below such experiments provide numbers whose role it is to test the Born rule as a hypothesis. This is justified by the following sampling theorem (strong law of large numbers): for any (measurable) subset $\Delta \subset \sigma(a)$ and any sequence $\left(x_{n}\right) \in \sigma(a)^{\mathbb{N}}$ we have $\mu_{a}^{\infty}$-almost surely:

$$
\lim _{N \rightarrow \infty} \frac{1}{N}\left(1_{\Delta}\left(x_{1}\right)+\cdots+1_{\Delta}\left(x_{N}\right)\right)=\mu_{a}(\Delta) .
$$

Proof of Theorem 4.1. Let $a=a^{*} \in B(H)$, where $H$ is a Hilbert space and $B(H)$ is the algebra of all bounded operators on $H$, and let $\sigma(a)$ be the spectrum of $a$. For simplicity (and since this is enough for our applications, where $H=\mathbb{C}^{2}$ ) I assume $\operatorname{dim}(H)<\infty$, so that $\sigma(a)$ simply consists of the eigenvalues $\lambda_{i}$ of $a$ (which may be degenerate). Let us first consider a finite number $N$ of identical measurements of $a$ (a "run"). The first option in the theorem corresponds to a simultaneous measurement of the commuting operators

$$
\begin{gathered}
a_{1}=a \otimes 1_{H} \otimes \cdots \otimes 1_{H} ; \\
\quad \cdots \\
a_{N}=1_{H} \otimes \cdots \otimes 1_{H} \otimes a,
\end{gathered}
$$

all defined on the $N$-fold tensor product $H^{N} \equiv H^{\otimes N}$ of $H$ with itself 31 To put this in a broader perspective, consider any set $\left(a_{1}, \ldots, a_{N}\right) \equiv \underline{a}$ of commuting operators on any Hilbert space $K$ (of which (A.3) - (A.4) is obviously a special case with $K=H^{N}$ ). These operators have a joint spectrum $\sigma(\underline{a})$, whose elements are the joint eigenvalues $\underline{\lambda}=\left(\lambda_{1}, \ldots, \lambda_{N}\right)$, defined by the property that there exists a nonzero joint eigenvector $\psi \in K$ such that $a_{i} \psi=\lambda_{i} \psi$ for all $i=1, \ldots, N$; clearly,

$$
\sigma(\underline{a})=\left\{\underline{\lambda} \in \sigma\left(a_{1}\right) \times \cdots \times \sigma\left(a_{n}\right) \mid e_{\underline{\lambda}} \equiv e_{\lambda_{1}}^{(1)} \cdots e_{\lambda_{n}}^{(n)} \neq 0\right\} \subseteq \sigma\left(a_{1}\right) \times \cdots \times \sigma\left(a_{N}\right),
$$

\footnotetext{
${ }^{30}$ Here a state $\omega$ is a positive normalized linear functional on $B(H)$, as in the $\mathrm{C}^{*}$-algebraic approach to quantum mechanics (Haag, 1992; Landsman, 2017). One may think of expectation values $\omega(a)=\operatorname{Tr}(\rho a)$, where $\rho$ is a density operator on $H$, with the special case $\omega(a)=\langle\psi, a \psi\rangle$, where $\psi \in H$ is a unit vector. For a proof of Theorem A.1 see e.g. Landsman (2017), §4.1, Corollary 4.4.

${ }^{31}$ This can even be replaced by a single measurement, see Landsman (2017), Corollary A.20.
} 
where $e_{\lambda_{i}}^{(i)}$ is the spectral projection of $a_{i}$ on the eigenspace for the eigenvalue $\lambda_{i} \in \sigma\left(a_{i}\right)$. Von Neumann's Born rule for the probability of finding $\underline{\lambda} \in \sigma(\underline{a})$ then simply reads

$$
p_{\underline{a}}(\underline{\lambda})=\omega\left(e_{\underline{\lambda}}\right),
$$

where $\omega$ is the state on $B(K)$ with respect to which the Born probability is defined 32 If $\operatorname{dim}(K)<\infty$, as I assume, we always have $\omega(a)=\operatorname{Tr}(\rho a)$ for some density operator $\rho$, and for a general Hilbert space $K$ this is the case iff the state $\omega$ is normal on $B(K)$. For (normal) pure states we have $\rho=|\psi\rangle\langle\psi|$ for some unit vector $\psi \in K$, in which case

$$
p_{\underline{a}}(\underline{\lambda})=\left\langle\psi, e_{\underline{\lambda}} \psi\right\rangle .
$$

The Born rule (A.6) is similar to the single-operator case (Landsman, 2017, §4.1) 33 the continuous functional calculus gives a Gelfand isomorphism of commutative $\mathrm{C}^{*}$-algebras

$$
C^{*}\left(\underline{a}, 1_{K}\right) \cong C(\sigma(\underline{a})),
$$

under which the restriction of the state $\omega$, originally defined on $B(K)$, to its commutative $\mathrm{C}^{*}$-subalgebra $C^{*}(\underline{a})$ defines a probability measure $\mu_{\underline{a}}$ on the joint spectrum $\sigma(\underline{a})$ via the Riesz isomorphism. This is the Born measure, whose probabilities are given by (A.6). For the case (A.3) - (A.4) we have equality in (A.5); since in that case $\sigma\left(a_{i}\right)=\sigma(a)$, we obtain

$$
\sigma(\underline{a})=\sigma(a)^{N},
$$

and therefore, for all $\lambda_{i} \in \sigma(a)$ and states $\omega$ on $B\left(H^{N}\right)$, the Born rule (A.6) becomes

$$
p_{\underline{a}}\left(\lambda_{1}, \ldots, \lambda_{N}\right)=\omega\left(e_{\lambda_{1}} \otimes \cdots \otimes e_{\lambda_{N}}\right) .
$$

Now take a state $\omega_{1}$ on $B(H)$. Reflecting the idea that $\omega$ is the state on $B\left(H^{N}\right)$ in which $N$ independent measurements of $a \in B(H)$ in the state $\omega_{1}$ are carried out, choose

$$
\omega=\omega_{1}^{N}
$$

the state on $B\left(H^{N}\right)$ defined by linear extension of its action on elementary tensors:

$$
\omega_{1}^{N}\left(b_{1} \otimes \cdots \otimes b_{n}\right)=\omega_{1}\left(b_{1}\right) \cdots \omega_{N}\left(b_{N}\right) .
$$

It follows that

$$
\omega^{N}\left(e_{\lambda_{1}} \otimes \cdots \otimes e_{\lambda_{N}}\right)=\omega_{1}\left(e_{\lambda_{1}}\right) \cdots \omega_{1}\left(e_{\lambda_{N}}\right)=p_{a}\left(\lambda_{1}\right) \cdots p_{a}\left(\lambda_{N}\right)
$$

so that the joint probability of the outcome $\left(\lambda_{1}, \ldots, \lambda_{N}\right) \in \sigma(\underline{a})$ is simply

$$
p_{\vec{a}}\left(\lambda_{1}, \ldots, \lambda_{N}\right)=p_{a}\left(\lambda_{1}\right) \cdots p_{a}\left(\lambda_{N}\right) .
$$

Since these are precisely the probabilities for option 2 (i.e. the Bernoulli process), i.e.,

$$
\mu_{\underline{a}}=\mu_{a}^{N},
$$

this proves the claim for $N<\infty$. To describe the limit $N \rightarrow \infty$, let $B$ be any $\mathrm{C}^{*}$-algebra with unit $1_{B}$; below I take $B=B(H), B=C^{*}\left(a, 1_{H}\right)$, or $B=C(\sigma(a))$. We now take

$$
A_{N}=B^{\otimes N}
$$

\footnotetext{
${ }^{32}$ The uses of states themselves may be justified by Gleason's theorem (Landsman, 2017, $\S \S 2,7,4.4$ ).

${ }^{33}$ The Born rule for commuting operators follows from the single operator case (Landsman, 2017, §2.5).
} 
the $N$-fold tensor product of $B$ with itself 34 The special cases above may be rewritten as

$$
\begin{aligned}
B(H)^{\otimes N} & \cong B\left(H^{N}\right) ; \\
C^{*}\left(a, 1_{H}\right)^{\otimes N} & \cong C^{*}\left(a_{1}, \ldots, a_{N}, 1_{H^{N}}\right) ; \\
C(\sigma(a))^{\otimes N} & \cong C(\sigma(a) \times \cdots \times \sigma(a)),
\end{aligned}
$$

with $N$ copies of $H$ and $\sigma(a)$, respectively, and in (A.18) the $a_{i}$ are given by (A.3) (A.4). We may then wonder if these algebras have a limit as $N \rightarrow \infty$. They do, but it is not unique and depends on the choice of observables, that is, of the infinite sequences $\mathbf{a}=\left(a_{1}, a_{2}, \ldots\right)$, with $a_{N} \in A_{N}$, that are supposed to have a limit. One possibility is to take sequences a for which there exists $M \in \mathbb{N}$ and $a_{M} \in A_{M}$ such that for each $N \geq M$,

$$
a_{N}=a_{M} \otimes 1_{B} \cdots \otimes 1_{B}
$$

with $N-M$ copies of $1_{B}$. On that choice, one obtains the infinite tensor product $B^{\otimes \infty}$, see Landsman (2017), §C.14. The limit of (A.17) in this sense is $B\left(H^{\otimes \infty}\right)$, where $H^{\otimes \infty}$ is von Neumann's 'complete' infinite tensor product of Hilbert spaces 35 in which $C^{*}\left(a, 1_{H}\right)^{\otimes \infty}$ is the $\mathrm{C}^{*}$-algebra generated by $\left(a_{1}, a_{2}, \ldots\right)$ and the unit on $H^{\otimes \infty}$. The limit of (A.19) is

$$
C(\sigma(a))^{\otimes \infty} \cong C\left(\sigma(a)^{\mathbb{N}}\right),
$$

where $\sigma(a)^{\mathbb{N}}$, which we previously saw as a measure space (as a special case of $X^{\mathbb{N}}$ for general compact Hausdorff spaces $X$ ), is now seen as a topological space with the product topology, in which it is compact 36 As in the finite case, we have an isomorphism

$$
C^{*}\left(a, 1_{H}\right)^{\otimes \infty} \cong C(\sigma(a))^{\otimes \infty},
$$

and hence, on the given identifications, we obtain an isomorphism of $\mathrm{C}^{*}$-algebras

$$
C^{*}\left(a_{1}, a_{2}, \ldots, 1_{H \otimes \infty}\right) \cong C\left(\sigma(a)^{\mathbb{N}}\right) .
$$

It follows from the definition of the infinite tensor products used here that each state $\omega_{1}$ on $B$ defines a state $\omega_{1}^{\infty}$ on $B^{\otimes \infty}$. Take $B=B(H)$ and restrict $\omega_{1}^{\infty}$, which a priori is a state on $B\left(H^{\otimes \infty}\right)$, to its commutative $\mathrm{C}^{*}$-subalgebra $C^{*}\left(a_{1}, a_{2}, \ldots, 1_{H^{\otimes \infty}}\right)$. The isomorphism (A.23) then gives a probability measure $\mu_{\underline{a}}$ on the compact space $\sigma(a)^{\mathbb{N}}$, where the label $\underline{a}$ now refers to the infinite set of commuting operators $\left(a_{1}, a_{2}, \ldots\right)$ on $H^{\otimes \infty}$. To compute this measure, I use (A.1) and the fact that by construction functions of the type

$$
f\left(\lambda_{1}, \lambda_{2}, \ldots\right)=f^{(N)}\left(\lambda_{1}, \ldots, \lambda_{N}\right),
$$

where $N<\infty$ and $f^{(N)} \in C\left(\sigma(a)^{N}\right)$, are dense in $C\left(\sigma(a)^{\mathbb{N}}\right)$ (with respect to the appropriate supremum-norm), and that in turn finite linear combinations of factorized functions $f^{(N)}\left(\lambda_{1}, \ldots, \lambda_{N}\right)=f_{1}\left(\lambda_{1}\right) \cdots f_{N}\left(\lambda_{N}\right)$ are dense in $C\left(\sigma(a)^{N}\right)$. It follows from this that

$$
\mu_{\underline{a}}=\mu_{a}^{\infty} .
$$

Since this generalizes (A.15) to $N=\infty$, the proof of Theorem 4.1 is finished.

\footnotetext{
${ }^{34}$ If $B$ is infinite-dimensional, for technical reasons the so-called projective tensor product should be used. ${ }^{35}$ See Landsman (2017), §8.4 for this approach. The details are unnecessary here.

${ }^{36} \mathrm{Cf}$. Tychonoff's theorem. The associated Borel structure is the one defined by the cylinder sets.
} 


\section{B 1-Randomness}

In what follows, the notion of 1-randomness, originally defined by Martin-Löf in the setting of constructive measure theory, will be explained through an equivalent definition in terms of Kolmogorov complexity 37 We assume basic familiarity with the notion of a computable function $f: \mathbb{N} \rightarrow \mathbb{N}$, which may be defined through recursion theory or Turing machines.

A string is a finite succession of bits (i.e. zeros and ones). The length of a string $\sigma$ is denoted by $|\sigma|$. The set of all strings of length $N$ is denoted by $\underline{2}^{N}$, where $\underline{2}=\{0,1\}$, and

$$
\underline{2}^{*}=\bigcup_{N \in \mathbb{N}} \underline{2}^{N}
$$

denotes the set of all strings. The Kolmogorov complexity $K(\sigma)$ of $\sigma \in \underline{2}^{*}$ is defined, roughly speaking, as the length of the shortest computer program that prints $\sigma$ and then halts. We then say, again roughly, that $\sigma$ is Kolmogorov random if this shortest program contains all of $\sigma$ in its code, i.e. if the shortest computable description of $\sigma$ is $\sigma$ itself.

To make this precise 38 fix some universal prefix-free Turing machine $U$, seen as performing a computation on input $\tau$ (in its prefix-free domain) with output $U(\tau)$, and define

$$
K(\sigma)=\min _{\tau \in \underline{2}^{*}}\{|\tau|: U(\tau)=\sigma\}
$$

The function $K: \underline{2}^{*} \rightarrow \mathbb{N}$ is uncomputable, but that doesn't mean it is ill-defined. The choice of $U$ affects $K(\sigma)$ up to a $\sigma$-independent constant, and to take this dependency into account we state certain results in terms of the "big-O" notation familiar from Analysis 39 For example, if $\sigma$ is easily computable, like the first $|\sigma|$ binary digits of $\pi$, then

$$
K(\sigma)=O(\log |\sigma|),
$$

with the logarithm in base 2 (as only the length of $\sigma$ counts). However, a random $\sigma$ has

$$
K(\sigma)=|\sigma|+O(\log |\sigma|) .
$$

We say that $\sigma$ is $c$-Kolmogorov random, for some $\sigma$-independent constant $c \in \mathbb{N}$, if

$$
K(\sigma) \geq|\sigma|-c .
$$

\footnotetext{
${ }^{37}$ For details see Volchan (2002), Terwijn (2016), Diaconis \& Skyrms (2018, Chapter 8), and Eagle (2019) for starters, technical surveys by Zvonkin \& Levin (1970), Muchnik et al. (1998), Downey et al. (2006), Grünwald \& Vitányi (2008), and Dasgupta (2011), and books by Calude (2002), Li \& Vitányi (2008), Nies (2009), and Downey \& Hirschfeldt (2010). For history see van Lambalgen (1987, 1996) and Li \& Vitányi (2008). For physical applications see e.g. Earman (1986), Svozil (1993, 2018), Calude (2004), Wolf (2015), Bendersky et al. (2016, 2017), Senno (2017), Baumeler et al. (2017), and Tadaki (2018, 2019).

${ }^{38} \mathrm{~A}$ Turing machine $T$ is prefix-free if its domain $D(T)$ consists of a prefix-free subset of $2^{*}$, i.e., if $\sigma \in D(T)$ then $\sigma \tau \notin D(T)$ for any $\sigma, \tau \in \underline{2}^{*}$, where $\sigma \tau$ is the concatenation of $\sigma$ and $\tau$ : if $T$ halts on input $\sigma$ then it does not halt on either any initial part or any extension of $\sigma$. The prefix-free version is only needed to correctly define randomness of sequences in terms of randomness of their initial parts, which is necessary to satisfy Earman's Principle: 'While idealizations are useful and, perhaps, even essential to progress in physics, a sound principle of interpretation would seem to be that no effect can be counted as a genuine physical effect if it disappears when the idealizations are removed.' See Earman (2004), p. 191. For finite strings $\sigma$ one may work with the plain Kolmogorov complexity $C(\sigma)$, defined as the length (in bits) of the shortest computer program (run on some fixed universal Turing machine $U$ ) that computes $\sigma$.

${ }^{39}$ Recall that $f(n)=O(g(n))$ iff there are constants $C$ and $N$ such that $|f(n)| \leq C|g(n)|$ for all $n \geq N$.
} 
Simple counting arguments show that as $|\sigma|=N$ gets large, the overwhelming majority of strings in $\underline{2}^{N}$ (and hence in $\underline{2}^{*}$ ) is c-random 40 The following theorem, which might be called Chaitin's first incompleteness theorem, therefore shows that randomness is elusive 41

Theorem B.1 For any sound mathematical theory $T$ containing enough arithmetic there is a constant $C \in \mathbb{N}$ such that $T$ cannot prove any sentence of the form $K(\sigma)>C$ (although infinitely many such sentences are true), and as such $T$ can only prove (Kolmogorov) randomness of finitely many strings (although infinitely many strings are in fact random).

The proof is quite complicated in its details but it is based on the existence of a computably enumerable (c.e.) list $\mathrm{T}=\left(\tau_{1}, \tau_{2}, \ldots\right)$ of the theorems of $T$, and on the fact that after Gödelian encoding by numbers, theorems of any given grammatical form can be computably searched for in this list and will eventually be found. In particular, there exists a program $P$ (running on the universal prefix-free Turing machine $U$ used to define $K(\cdot))$ such that $P(n)$ halts iff there exists a string $\sigma$ for which $K(\sigma)>n$ is a theorem of $T$. If there is such a theorem the output is $P(n)=\sigma$, where $\sigma$ appears in the first such theorem of the kind (according to the list $\mathrm{T}$ ). By definition of $K(\cdot)$, this means that

$$
K(\sigma) \leq|P|+|n| .
$$

Now suppose that no $C$ as in the above statement of the theorem exists. Then there is $n \in \mathbb{N}$ large enough that $n>|P|+|n|$ and there is a string $\sigma \in \underline{2}^{*}$ such that $T$ proves $K(\sigma)>n$. Since $T$ is sound this is actually true ${ }^{42}$ which gives a contradiction between

$$
K(\sigma)>n>|P|+|n| ; \quad K(\sigma) \leq|P|+|n| .
$$

Note that this proof shows that a proof in $T$ of $K(\sigma)>n$ (if true) would also identify $\sigma$.

As an idealization of a long (binary) string, a (binary) sequence $x=x_{1} x_{2} \cdots$ is an infinite succession of bits, i.e. $x \in \underline{2}^{\mathbb{N}}$, with finite truncations $x_{\mid N}=x_{1} \cdots x_{N} \in \underline{2}^{N}$ for each $N \in \mathbb{N}$. We then call $x$ Levin-Chaitin random if each truncation of $x$ is $c$-Kolmogorov random for some $c$, that is, if there exists $c \in \mathbb{N}$ such that $K\left(x_{\mid N}\right) \geq N-c$ for each $N \in \mathbb{N}$. Equivalently 43 a sequence $x$ is Levin-Chaitin random if eventually $K\left(x_{\mid N}\right)>>N$, in that

$$
\lim _{N \rightarrow \infty}\left(K\left(x_{\mid N}\right)-N\right)=\infty .
$$

Apart from having the same intuitive pull as Kolmogorov randomness (of strings), this definition gains from the fact that it is equivalent to two other appealing notions of randomness, namely patternlessness and unpredictability, both also defined computationally.

\footnotetext{
${ }^{40}$ It is easy to show that least $2^{N}-2^{N-c+1}+1$ strings $\sigma$ of length $|\sigma|=N$ are $c$-Kolmogorov random.

${ }^{41}$ Here "sound" means that all theorems proved by $T$ are true; this is a stronger assumption than consistency (in fact only the arithmetic fragment of $T$ needs to be sound). One may think of Peano Arithmetic (PA) or of Zermelo-Fraenkel set theory with the axiom of choice (ZFC). As in Gödel's theorems, one also assumes that $T$ is formalized as an axiomatic-deductive system in which proofs could in principle be carried out mechanically by a computer. The status of the true but unprovable sentences $K(\sigma)>C$ in Chaitin's theorem is similar to that of the sentence $G$ in Gödel's original proof of his first incompleteness theorem, which roughly speaking is an arithmetization of the statement "I cannot be proved in $T$ ": assuming soundness and hence consistency of $T$, one can prove $\mathrm{G}$ and $K(\sigma)>C$ in the usual interpretation of the arithmetic fragment of $T$ in the natural numbers $\mathbb{N}$. See Chaitin (1987) for his own presentation and analysis of his incompleteness theorem. Raatikainen (1998) also gives a detailed presentation of the theorem, including a critique of Chaitin's ideology. Incidentally, he shows that there even exists a $U$ with respect to which $K(\cdot)$ is defined such that $C=0$ in ZFC. See also Franzén (2005) and Gács (1989).

${ }^{42}$ The following contradiction can be made more dramatic by taking $n$ such that $n>>|P|+|n|$.

${ }^{43}$ See Calude (2002), Theorem 6.38 (attributed to Chaitin) for this equivalence.
} 
In view of these equivalences we simply call a Levin-Chaitin random sequence 1-random 44 A sequence $x \in \underline{k}^{\mathbb{N}}$ is Borel normal in base $k$ if each string $\sigma$ has frequency $k^{-|\sigma|}$ in $x$. Any hope of defining randomness as Borel normality in base 10 is blocked by Champernowne's number $0123456789101112131 \cdots$, which is Borel normal but clearly not random in any reasonable sense (this is also true in base 2). The decimal expansion of $\pi$ is also conjectured to be Borel normal in base 10 (with huge numerical support), although $\pi$ clearly is not random either. However, Borel normality seems a desirable property of truly random numbers on any good definition, and so we are fortunate to have:

Proposition B.2 A 1-random sequence is Borel normal (in base 2, but in fact in any base) and hence ("monkey typewriter theorem") contains any finite string infinitely often 45

Another desirable property comes from the following theorem due to Martin-Löf, in which $P$ is the 50-50 probability on $\{0,1\}$ and $P^{\infty}$ is the induced probability measure on $\underline{2}^{\mathbb{N}}$ :

Theorem B.3 With respect to $P^{\infty}$ almost every outcome sequence $x \in \underline{2}^{\mathbb{N}}$ is 1-random.

This implies that the 1-random sequences form an uncountable subset of $\underline{2}^{\mathbb{N}}, 46$ although topologically this subset is meagre (i.e. Baire first category) 47 Chaitin's (first) incompleteness theorem for (finite) strings has the following counterpart for (infinite) sequences:

Theorem B.4 If $x \in \underline{2}^{\mathbb{N}}$ is 1-random, then $Z F C$ (or any sufficiently comprehensive mathematical theory $T$ meant in Theorem (B.1) can compute only finite many digits of $x .48$

This clearly excludes defining a 1-random number by somehow listing its digits, but some can be described by a formula. One example is Chaitin's $\Omega$, or more precisely $\Omega_{U}, 49$ which is the halting probability of some fixed universal prefix-free Turing machine $U$, given by

$$
\Omega_{U}:=\sum_{\tau \in \underline{2}^{*} \mid U(\tau) \downarrow} 2^{-|\tau|} .
$$

\footnotetext{
${ }^{44}$ Any pattern in a sequence $x$ would make it compressible, but one has to define the notion of a pattern very carefully in a computational setting. This was accomplished by Martin-Löf in 1966, who defined a pattern as a specific kind of probability-zero subset $T$ of $\underline{2}^{\mathbb{N}}$ (called a "test") that can be computably approximated by subsets $T_{n} \subset \underline{2}^{\mathbb{N}}$ of increasingly small probability $2^{-n}$; if $x \in T$, then $x$ displays some pattern and it is patternless iff $x \notin T$ for all such tests. Martin-Löf's definition yields what usually called 1-randomness, in view of his use of so-called $\Sigma_{1}^{0}$ sets. See the textbooks Li \& Vitányi (2008), Calude (2002), Nies (2009), and Downey \& Hirschfeldt (2010) for the equivalences between Levin-Chaitin randomness (incompressibility), Martin-Löf randomness (patternlessless), and a third notion (unpredictability) that evolved from the work of von Mieses and Ville, finalized by Schnorr. The name Levin-Chaitin randomness, taken from Downey et al. (2006), is justified by its independent origin in Levin (1973) and Chaitin (1975).

${ }^{45}$ For details and proofs see Calude (2002), Corollary 6.32 in $\S 6.3$ and almost all of $\S 6.4$.

${ }^{46}$ To see this, use the measure-theoretic isomorphism between $\left(\underline{2}^{\mathbb{N}}, \Sigma_{K}, P^{\infty}\right)$ and $\left([0,1], \Sigma_{L}, d x\right)$, where $\Sigma_{K}$ is the "Kolmogorov" $\sigma$-algebra generated by the cylinder sets $[\bar{\sigma}]=\left\{x \in \underline{2}^{\mathbb{N}} \mid x_{|| \sigma \mid}=\sigma\right\}$, where $\sigma \in \underline{2}^{*}$, and $\Sigma_{K}$ is the "Lebesgue" $\sigma$-algebra generated by the open subsets of $[0,1]$. See also Nies $(2009), \S 1.8$.

${ }^{47}$ See Calude (2002), Theorem 6.63. Hence meagre subsets of [0,1] exist with unit Lebesgue measure!

${ }^{48}$ More precisely, only finitely many true statements of the form: 'the $n$ 'th bit $x_{n}$ of $x$ equals its actual value' (i.e. 0 or 1 ) are provable in $T$ (where a proof in $T$ may be seen as a computation, since one may algorithmically search for this proof in a list). See Calude (2002), Theorem 8.7, which is stated for Chaitin's $\Omega$ but whose proof holds for any 1-random sequence. Indeed, as pointed out to the author by Bas Terwijn, even more generally, ZFC (etc.) can only compute finitely many digits of any immune sequence (we say that a sequence $x \in \underline{2}^{\mathbb{N}}$ is immune if the corresponding subset $S \subset \mathbb{N}$ (i.e. $1_{S}=x$ ) contains no infinite c.e. subset), and by (for example) Corollary 6.42 in Calude (2002) any 1-random sequence is immune.

${ }^{49}$ There exists a $U$ for which not a single digit of $\Omega_{U}$ can be known, see Calude (2002), Theorem 8.11.
} 


\section{Bell's theorem and free will theorem}

In support of the analysis of hidden variable theories in the main text, this appendix reviews Bell's (1964) theorem and the free will theorem, streamlining earlier expositions (Cator \& Landsman, 2014; Landsman, 2017, Chapter 6) and leaving out proofs and other adornments 50 In the specific context of 't Hooft's theory (where the measurement settings are determined by the hidden state) and Bohmian mechanics (where they are not, as in the original formulation of Bell's theorem and in most hidden variable theories) an advantage of my approach is that both free (uncorrelated) und correlated settings fall within its scope; the former are distinguished from the latter by an independence assumption 51

As a warm-up I start with a version of the Kochen-Specker theorem, whose logical form is very similar to Bell's (1964) theorem and the free will theorem, as follows:

Theorem C.1 Determinism, QM, non-contextuality, and free choice are contradictory.

Of course, this unusual formulation hinges on the precise meaning of these terms.

- determinism is the conjunction of the following two assumptions.

1. There is a state space $X$ with associated functions $A: X \rightarrow S$ and $L: X \rightarrow O$, where $S$ is the set of all possible measurement settings Alice can choose from, namely a suitable finite set of orthonormal bases of $\mathbb{R}^{3}$ (11 well-chosen bases will do to arrive at a contradiction) 52 and $O$ is some set of possible measurement outcomes. Thus some $x \in X$ determines both Alice's setting $a=A(x)$ and her outcome $\alpha=L(x)$.

2. There exists some set $\Lambda$ and an additional function $H: X \rightarrow \Lambda$ such that

$$
L=L(A, H),
$$

in the sense that for each $x \in X$ one has $L(x)=\hat{L}(A(x), H(x))$ for a certain function $\hat{L}: S \times \Lambda \rightarrow O$. This self-explanatory assumption just states that each measurement outcome $L(x)=\hat{L}(a, \lambda)$ is determined by the measurement setting $a=A(x)$ and the "hidden" variable or state $\lambda=H(x)$ of the particle undergoing measurement.

- QM fixes $O=\{(0,1,1),(1,0,1),(1,1,0)\}$, which is a non-probabilistic fact of quantum mechanics with overwhelming (though indirect) experimental support.

- non-contextuality stipulates that the function $\hat{L}$ just introduced take the form

$$
\hat{L}\left(\left(\vec{e}_{1}, \vec{e}_{2}, \vec{e}_{3}\right), \lambda\right)=\left(\tilde{L}\left(\vec{e}_{1}, \lambda\right), \tilde{L}\left(\vec{e}_{2}, \lambda\right), \tilde{L}\left(\vec{e}_{3}, \lambda\right)\right),
$$

\footnotetext{
${ }^{50}$ The original reference for Bell's theorem is Bell (1964); see further footnote 6, and in the context of this appendix also Esfeld (2015) and Sen \& Valentini (2020) are relevant. The free will theorem originates in Heywood \& Redhead (1983), followed by Stairs (1983), Brown \& Svetlichny (1990), Clifton (1993), and, as name-givers, Conway \& Kochen (2009). Both theorems can and have been presented and interpreted in many different ways, of which we choose the one that is relevant for the general discussion on randomness in the main body of the paper. This appendix is taken almost verbatim from Landsman (2020).

${ }^{51}$ This addresses a problem Bell faced even according to some of his most ardent supporters (Norsen, 2009; Seevinck \& Uffink, 2011), namely the tension between the idea that the hidden variables (in the pertinent causal past) should on the one hand include all ontological information relevant to the experiment, but on the other hand should leave Alice and Bob free to choose any settings they like. Whatever its ultimate fate, 't Hooft's staunch determinism has drawn attention to issues like this, as has the free will theorem.

${ }^{52}$ If her setting is a basis $\left(\vec{e}_{1}, \vec{e}_{2}, \vec{e}_{3}\right)$, Alice measures the quantities $\left(J_{\vec{e}_{1}}^{2}, J_{\vec{e}_{2}}^{2}, J_{\vec{e}_{3}}^{2}\right)$, where $J_{\vec{e}_{1}}=\left\langle\vec{J}, \vec{e}_{i}\right\rangle$ is the component of the angular momentum operator $\vec{J}$ of a massive spin-1 particle in the direction $\vec{e}_{i}$.
} 
for a single function $\tilde{L}: S^{2} \times \Lambda \rightarrow\{0,1\}$ that also satisfies $\tilde{L}(-\vec{e}, \lambda)=\tilde{L}(\vec{e}, \lambda) 53$

- free choice finally states that the following function is surjective:

$$
A \times H: X \rightarrow S \times \Lambda ; \quad x \mapsto(A(x), H(x)) .
$$

In other words, for each $(a, \lambda) \in S \times \Lambda$ there is an $x \in X$ for which $A(x)=a$ and $H(x)=\lambda$. This makes $A$ and $H$ "independent" (or: makes $a$ and $\lambda$ free variables).

See Landsman (2017), §6.2 for a proof of the Kochen-Specker theorem in this language 54

Bell's (1964) theorem and the free will theorem both take a similar generic form, namely:

Theorem C.2 Determinism, QM, local contextuality, and free choice, are contradictory.

Once again, I have to explain what these terms exactly mean in the given context.

- determinism is a straightforward adaptation of the above meaning to the bipartite "Alice and Bob" setting. Thus we have a state space $X$ with associated functions

$$
A: X \rightarrow S ; \quad B: X \rightarrow S ; \quad L: X \rightarrow O \quad R: X \rightarrow O,
$$

where $S$, the set of all possible measurement settings Alice and Bob can each choose from, differs a bit between the two theorems: for the free will theorem it is the same as for the Kochen-Specker theorem above, as is the set $O$ of possible measurement outcomes, whereas for Bell's theorem (in which Alice and Bob each measure a 2-level system), $S$ is some finite set of angles (three is enough), and $O=\{0,1\}$.

- In the free will case, these functions and the state $x \in X$ determine both the settings $a=A(x)$ and $b=B(x)$ of a measurement and its outcomes $\alpha=L(x)$ and $\beta=R(x)$ for Alice on the Left and for Bob on the Right, respectively.

- All of this is also true in the Bell case, but since his theorem relies on impossible measurement statistics (as opposed to impossible individual outcomes), one in addition assumes a probability measure $\mu$ on $X 55$

Furthermore, there exists some set $\Lambda$ and some function $H: X \rightarrow \Lambda$ such that

$$
L=L(A, B, H) ; \quad R=R(A, B, H),
$$

in the sense that for each $x \in X$ one has functional relationships

$$
L(x)=\hat{L}(A(x), B(x), H(x)) ; \quad R(x)=\hat{R}(A(x), B(x), H(x)),
$$

for certain functions $\hat{L}: S \times S \times \Lambda \rightarrow O$ and $\hat{R}: S \times S \times \Lambda \rightarrow O$.

\footnotetext{
${ }^{53}$ Here $S^{2}=\left\{(x, y, z) \in \mathbb{R}^{3} \mid x^{2}+y^{2}+z^{2}=1\right\}$ is the 2-sphere, seen as the space of unit vectors in $\mathbb{R}^{3}$. Eq. (C.2) means that the outcome of Alice's measurement of $J_{\vec{e}_{i}}^{2}$ is independent of the "context" $\left(J_{\vec{e}_{1}}^{2}, J_{\vec{e}_{2}}^{2}, J_{\vec{e}_{3}}^{2}\right)$; she might as well measure $J_{\vec{e}_{i}}^{2}$ by itself. The last equation is trivial, since $\left(J_{-\vec{e}_{i}}\right)^{2}=\left(J_{\vec{e}_{i}}\right)^{2}$.

${ }^{54}$ The assumptions imply the existence of a coloring $C_{\lambda}: \mathcal{P} \rightarrow\{0,1\}$ of $\mathbb{R}^{3}$, where $\mathcal{P} \subset S^{2}$ consist of all unit vectors contained in all bases in $S$, and $\lambda$ "goes along for a free ride". A coloring of $\mathbb{R}^{3}$ is a function $C: \mathcal{P} \rightarrow\{0,1\}$ such that for any set $\left\{e_{1}, e_{2}, e_{3}\right\}$ in $\mathcal{P}$ with $e_{i} e_{j}=\delta_{i j} 1_{3}$ and $e_{1}+e_{2}+e_{3}=1_{3}$ where $1_{3}$ is the $3 \times 3$ unit matrix) there is exactly one $e_{i}$ for which $C\left(e_{i}\right)=1$. Indeed, one finds $C_{\lambda}(\vec{e})=\tilde{L}(\vec{e}, \lambda)$. The key to the proof of Kochen-Specker is that on a suitable choice of the set $S$ such a coloring cannot exist.

${ }^{55}$ The existence of $\mu$ is of course predicated on $X$ being a measure space with corresponding $\sigma$-algebra of measurable subsets, with respect to which all functions in (C.4 and below are measurable.
} 
- QM reflects elementary quantum mechanics of correlated 2-level and 3-level quantum systems for the Bell and the free will cases, respectively, as follows 56

- In the free will theorem, $O=\{(0,1,1),(1,0,1),(1,1,0)\}$ is the same as for the Kochen-Specker theorem. In addition perfect correlation obtains: if $a=$ $\left(\vec{e}_{1}, \vec{e}_{2}, \vec{e}_{3}\right)$ is Alice's orthonormal basis and $b=\left(\vec{f}_{1}, \vec{f}_{2}, \overrightarrow{f_{3}}\right)$ is Bob's, one has

$$
\vec{e}_{i}=\vec{f}_{j} \Rightarrow \hat{L}_{i}(a, b, z)=\hat{R}_{j}(a, b, z),
$$

where $\hat{L}_{i}, \hat{R}_{j}: S \times S \times \Lambda \rightarrow\{0,1\}$ are the components of $\hat{L}$ and $\hat{R}$, respectively. Finally 57 if $\left(a^{\prime}, b^{\prime}\right)$ differs from $(a, b)$ by changing the sign of any basis vector,

$$
\hat{L}\left(a^{\prime}, b^{\prime}, \lambda\right)=\hat{L}(a, b, \lambda) ; \quad \hat{R}\left(a^{\prime}, b^{\prime}, \lambda\right)=\hat{R}(a, b, \lambda) .
$$

- In Bell's theorem, $O=\{0,1\}$, and the statistics for the experiment is reproduced as conditional joint probabilities given by the measure $\mu$ through

$$
P(L \neq R \mid A=a, B=b)=\sin ^{2}(a-b) .
$$

- local contextuality, which replaces and weakens non-contextuality, means that

$$
L(A, B, H)=L(A, H) ; \quad R(A, B, H)=G(B, H) .
$$

In words: Alice's outcome given $\lambda$ does not depend on Bob's setting, and vice versa.

- free choice is an independence assumption that looks differently for both theorems:

- In the free will theorem it means that each $(a, b, \lambda) \in S \times S \times \Lambda$ is possible in that there is an $x \in X$ for which $A(x)=a, B(x)=b$, and $H(x)=\lambda$.

- In Bell's theorem, $(A, B, H)$ are probabilistically independent relative to $\mu .58$

This concludes the joint statement of the free will theorem and Bell's (1964) theorem in the form we need for the main text. The former is proved by reduction to the Kochen-Specken theorem, whilst the latter follows by reduction to the usual version of Bell's theorem via the free choice assumption; see Landsman (2017), Chapter 6 for details.

For our purposes these theorems are equivalent, despite subtle differences in their assumptions. Bell's theorem is much more robust in that it does not rely on perfect correlations (which are hard to realize experimentally), and in addition it requires almost no input from quantum theory. On the other hand, Bell's theorem uses probability theory in a highly nontrivial way: like the hidden variable theories it is supposed to exclude it relies on the possibility of fair sampling of the probability measure $\mu$. The factorization condition defining probabilistic independence passes this requirement of fair sampling on to both the hidden variable and the settings, which brings us back to the main text.

Different parties may now be identified by the assumption they drop: Copenhagen quantum mechanics rejects determinism, Valentini (2019) rejects the Born rule and hence QM, Bohmians rejects local contextuality, and finally 't Hooft rejects free choice. However, as we argue in the main text, even the latter two camps do not really have a deterministic theory underneath quantum mechanics because of their need to randomly sample the probability measure they must use to recover the predictions of quantum mechanics.

\footnotetext{
${ }^{56}$ In Bell's theorem quantum theory can be replaced by experimental support (Hensen et al., 2015).

${ }^{57} \mathrm{As}$ in Kochen-Specker, this is because Alice \& Bob measure squares of (spin-1) angular momenta.

${ }^{58}$ By definition, this also implies that the pairs $(A, B),(A, H)$, and $(B, H)$ are also independent.
} 


\section{References}

[1] Baumeler, A., Bédard, C.A., Brassard, G., Wolf, S. (2017). Kolmogorov amplification from Bell correlation, IEEE International Symposium on Information Theory (ISIT). doi:10.1109/ISIT.2017.8006790.

[2] Bell, J.S. (1964). On the Einstein-Podolsky-Rosen Paradox, Physics 1, 195-200.

[3] Bell, J.S. (1966). On the problem of hidden variables in quantum mechanics, Reviews of Modern Physics 38, 447-452.

[4] Bendersky, A., de la Torre, G., Senno, G., Figueira, S., Acín, A. (2016). Algorithmic pseudorandomness in quantum setups, Physical Review Letters 116, 230402.

[5] Bendersky, A., Senno, G., de la Torre, G., Figueira, S., Acín, A. (2017). Nonsignaling deterministic models for nonlocal correlations have to be incomputable, Physical Review Letters $118,130401$.

[6] Bohm, D. (1952). A suggested interpretation of the quantum theory in terms of 'hidden' variables, I, II, Physical Review 85, 166-179, 180-193.

[7] Bohr, N. 91935). Can quantum-mechanical description of physical reality be considered complete?, Physical Review 48, 696-702.

[8] Born, M. (1926). Zur Quantenmechanik der Stoßvorgänge, Zeitschrift für Physik 37, 863-867.

[9] Breuer, T. (2001). Von Neumann, Gödel and quantum incompleteness, John von Neumann and the Foundations of Quantum Physics, eds. Rédei, M., Stöltzner, M., pp. 75-82 (Kluwer).

[10] Broglie, L. de (1928). La nouvelle dynamique des quanta, Electrons et Photons: Rapports et Discussions du Cinquième Conseil de Physique, pp. 105-132 (Gauthier-Villars).

[11] Brown, H., Svetlichny, G. (1990). Nonlocality and Gleason's lemma. Part I. deterministic theories, Foundations of Physics 20, 1379-1387.

[12] Brown, H., Timpson, C.G. (2014). Bell on Bell's theorem: The changing face of nonlocality, $\backslash$ protect \vrule width0pt \protect \href $\{$ http://arxiv.org/abs/1501.03521\}\{arXiv:1501.03521\}.

[13] Bub, J. (2011). Is von Neumann's 'no hidden variables' proof silly?, Deep Beauty: Mathematical Innovation and the Search for Underlying Intelligibility in the Quantum World, ed. Halvorson, H., pp. 393-408 (Cambridge University Press).

[14] Butterfield, J. (1992). Bell's theorem: what it takes, The British Journal for the Philosophy of Science 43, 41-83.

[15] Calude, C.S. (2002). Information and Randomness: An Algorithmic Perspective, 2nd Edition (Springer).

[16] Calude, C.S. (2004). Algorithmic randomness, quantum physics, and incompleteness, Lecture Notes in Computer Science 3354, 1-17.

[17] Cator, E., Landsman, N.P. (2014). Constraints on determinism: Bell versus Conway-Kochen, Foundations of Physics 44, 781-791.

[18] Clifton, R. (1993). Getting contextual and nonlocal elements-of-reality the easy way, American Journal of Physics 61, 443-447.

[19] Chaitin, G.J. (1975). A theory of program size formally identical to information theory, Journal of the Association for Computing Machinery 22, 329-340.

[20] Chaitin, G.J. (1987). Information, Randomness 6 Incompleteness: Papers on Algorithmic Information Theory (World Scientific).

[21] Conway, J.H., Kochen, S. (2009). The Strong Free Will Theorem, Notices of the American Mathematical Society 56, 226-232. 
[22] Corry, L. (2004). David Hilbert and the Axiomatization of Physics (1898-1918): From Grundlagen der Geometrie to Grundlagen der Physik (Kluwer).

[23] Crull, E., Bacciagaluppi, G. (2016). Grete Hermann-Between Physics and Philosophy (Springer).

[24] Dasgupta, A. (2011). Mathematical Foundations of Randomness, Handbook of the Philosophy of Science. Volume 7: Philosophy of Statistics, eds. Bandyopadhyay, P.S., Forster, M.R., pp. 641-710 (North-Holland/Elsevier).

[25] Diaconis, P., Skyrms, B. (2018). Ten Great Ideas About Chance (Princeton University Press).

[26] Dieks, D. (2016). Von Neumann's impossibility proof: Mathematics in the service of rhetorics, Studies in History and Philosophy of Modern Physics 60, 136-148.

[27] Downey, R., Hirschfeldt, D.R. (2010). Algorithmic Randomness and Complexity (Springer).

[28] Downey, R., Hirschfeldt, D.R., Nies, A., Terwijn, S.A. (2006). Calibrating randomness, Bulletin of Symbolic Logic 12, 411-491.

[29] Dürr, D., Goldstein, S., Zanghi, N. (1992). Quantum equilibrium and the origin of absolute uncertainty, Journal of Statistical Physics 67, 843-907.

[30] Eagle, A. (2019). Chance versus Randomness, The Stanford Encyclopedia of Philosophy, $\mathrm{URL}=$ https://plato.stanford.edu/archives/spr2019/entries/chance-randomness/.

[31] Earman, J. (1986). A Primer on Determinism. Dordrecht: D. Reidel.

[32] Earman, J. (2004). Curie's Principle and spontaneous symmetry breaking, International Studies in Philosophy of Science 18, 173-198.

[33] Einstein, A., Podolsky, B., Rosen, N. (1935). Can quantum-mechanical description of physical reality be considered complete?, Physical Review 47, 777-780.

[34] Esfeld, M. (2015). Bell's Theorem and the issue of determinism and indeterminism, Foundations of Physics 45, 471-482.

[35] Franzén, T. (2005). Gödel's Theorem: An Incomplete Guide to its Use and Abuse (AK Peters).

[36] Gács, P. (1989). Review of "Algorithmic Information Theory" by Gregory J. Chaitin, The Journal of Symbolic Logic 54, 624-627.

[37] Gödel, K. (1931). Über formal unentscheidbare Sätze der Principia Mathematica und verwandter Systeme I, Monatshefte für Mathematik und Physik 38, 173-198.

[38] Goldstein, S. (2017). Bohmian Mechanics, The Stanford Encyclopedia of Philosophy, https://plato.stanford.edu/archives/sum2017/entries/qm-bohm/.

[39] Greenstein, G. (2019). Quantum Strangeness: Wrestling with Bell's Theorem and the Ultimate Nature of Reality (The MIT Press).

[40] Grünwald, P.D., Vitányi, P.M.B. (2008). Algorithmic information theory, Handbook of the Philosophy of Information, eds. Adriaans, P., van Benthem, J., pp. 281-320 (Elsevier).

[41] Haag, R. (1992). Local Quantum Physics: Fields, Particles, Algebras (Springer).

[42] Hacking, I. (1990). The Taming of Chance (Cambridge University Press).

[43] Hensen, B. et al (2015). Experimental loophole-free violation of a Bell inequality using entangled electron spins separated by $1.3 \mathrm{~km}$, Nature 526, 682-686.

[44] Heywood, P., Redhead, M. (1983). Nonlocality and the Kochen-Specker paradox, Foundations of Physics 13, 481-499.

[45] Hilbert, D. (1902). Mathematical Problems. Lecture delivered before the International Congress of Mathematicians at Paris in 1900, Bulletin of the American Mathematical Society 8, 437-479. Translated from Göttinger Nachrichten, 1900, pp. 253-297. 
[46] Hooft, G. 't (2016). The Cellular Automaton Interpretation of Quantum Mechanics (Springer Open). https://www . springer.com/gp/book/9783319412849.

[47] Kline, M. (1980). Mathematics: The Loss of Certainty (Oxford University Press).

[48] Kochen, S., Specker, E. (1967). The problem of hidden variables in quantum mechanics, Journal of Mathematics and Mechanics 17, 59-87.

[49] Kolmogorov, A.N. (1933). Grundbegriffe de Wahrscheinlichkeitsrechnung (Springer-Verlag).

[50] Lambalgen, M. van (1987). Random Sequences. PhD Thesis, University of Amsterdam, https://www . academia.edu/23899015/RANDOM_SEQUENCES.

[51] Lambalgen, M. van (1996). Randomness and foundations of probability: Von Mises' axiomatisation of random sequences, Statistics, Probability and Game Theory: Papers in Honour of David Blackwell, IMS Lecture Notes-Monograph Series, Vol. 30, pp. 347-367.

[52] Landsman, K. (2017). Foundations of Quantum Theory: From Classical Concepts to Operator Algebras (Springer Open). https://www.springer.com/gp/book/9783319517766.

[53] Landsman, K. (2020). Randomness? What randomness?, Foundations of Physics 50, 61-104.

[54] Landsman, K. (2021). Quantum theory and functional analysis, Oxford Handbook of the History of Interpretations and Foundations of Quantum Mechanics, ed. O. Freire, to appear (Oxford University Press).

[55] Levin, L.A. (1973). On the notion of a random sequence, Soviet Mathematics-Doklady 14, 1413-1416.

[56] Li, M., Vitányi, P.M.B. (2008). An Introduction to Kolmogorov Complexity and Its Applications. Third Edition (Springer).

[57] Mehra, J., Rechenberg, H. (2000). The Historical Development of Quantum Theory. Vol. 6: The Completion of Quantum Mechanics 1926-1941. Part 1 (Springer).

[58] Mises, R. von (1919). Grundlagen der Wahrscheinlichkeitsrechnung, Mathematische Zeitschrift $5,52-99$.

[59] Montague, R. (1974). Deterministic theories, Formal Philosophy: Selected Papers of Richard Montague, ed. Thomason, R.H., pp. 303-360 (Yale University Press).

[60] Moschovakis, J.R. (2016). Iterated definability, lawless sequences and Brouwer's continuum. Gödel's Disjunction, eds. Horsten, L., Welch, P., pp. 92-107 (Oxford University Press).

[61] Muchnik, A.A., Semenov, A.L., Uspensky, V.A. (1998). Mathematical metaphysics of randomness, Theoretical Computer Science 207, 263-317.

[62] Neumann, J. von (1932). Mathematische Grundlagen der Quantenmechanik (SpringerVerlag). English translation: Mathematical Foundations of Quantum Mechanics (Princeton University Press, 1955).

[63] Nies, A. (2009). Computability and Randomness (Oxford University Press).

[64] Norsen, T. (2009). Local causality and completeness: Bell vs. Jarrett, Foundations of Physics $39,273-294$.

[65] Pour-El, M.B., \& Richards, J.I. (2016). Computability in Analysis and Physics (Cambridge University Press).

[66] Raatikainen, P. (1998). On interpreting Chaitin's incompleteness theorem, Journal of Philosophical Logic 27, 569-586.

[67] Rédei, M., Stöltzner, eds. (2001). John von Neumann and the Foundations of Quantum Physics. Dordrecht: Kluwer.

[68] Redhead, M. (1989). Incompleteness, Nonlocality, and Realism (Oxford University Press). 
[69] Renn, J., ed. (2007). The Genesis of General Relativity, Vol. 4 (Springer).

[70] Scriven, M. (1957). The present status of determinism in physics, Journal of Philosophy 54, $727-741$.

[71] Seevinck, M.P., Uffink, J. (2011). Not throwing out the baby with the bathwater: Bell's condition of local causality mathematically 'sharp and clean', Explanation, Prediction, and Confirmation 2, 425-450.

[72] Sen, I., Valentini, A. (2020a). Superdeterministic hidden-variables models I: nonequilibrium and signalling, \protect \vrule widthOpt\protect \href\{http://arxiv.org/abs/2003.11989\}\{arXiv:200 II: conspiracy, \protect \vrule widthOpt \protect \href \{http://arxiv.org/abs/2003.12195\}\{arXiv:200

[73] Senno, G. (2017). A Computer-Theoretic Outlook on Foundations of Quantum Information, Ph.D. Thesis, Universidad de Buenos Aires.

[74] Sieg, W. (2013). Hilbert's Programs and Beyond (Oxford University Press).

[75] Smith, P. (2013). An Introduction to Gödel's Theorems (Cambridge University Press).

[76] Stairs, A. (1983). Quantum logic, realism, and value definiteness, Philosophy of Science 50, $578-602$.

[77] Svozil, K. (1993). Randomness 83 Undecidability in Physics (World Scientific).

[78] Svozil, K. (2018). Physical (A)Causality: determinism, Randomness and Uncaused Events (Springer Open). https://www. springer.com/gp/book/9783319708140.

[79] Svozil, K., Calude, C.S., Stay, M.A. (2005). From Heisenberg to Gödel via Chaitin, International Journal of Theoretical Physics 44, 1053-1065.

[80] Szangolies, J. (2018). Epistemic horizons and the foundations of quantum mechanics, arXiv: 1805.10668.

[81] Tadaki, K. (2018). A refinement of quantum mechanics by algorithmic randomness, arXiv: 1804.10174.

[82] Tadaki, K. (2019). A Statistical Mechanical Interpretation of Algorithmic Information Theory (Springer).

[83] Tait, W. (2005). The Provenance of Pure Reason: Essays in the Philosophy of Mathematics and Its History (Oxford University Press).

[84] Tapp, C. (2013). An den Grenzen des Endlichen: Das Hilbertprogramm (Springer).

[85] Terwijn, S.A. (2016). The Mathematical Foundations of Randomness, The Challenge of Chance: A Multidisciplinary Approach from Science and the Humanities, eds. Landsman, K., van Wolde, E., pp. 49-66 (Springer), https://www. springer.com/gp/book/9783319262987.

[86] Valentini, A. (2019). Foundations of statistical mechanics and the status of the Born rule in de Broglie-Bohm pilot-wave theory, arXiv:1906.10761.

[87] Volchan, S.B. (2002). What is a random sequence?, The American Mathematical Monthly 109, 46-63.

[88] Werner, R.F., Wolf, M.M. (2001). Bell inequalities and entanglement, Quantum Information \& Computation 1, 1-25.

[89] Wiseman, H.M. (2014). The two Bell's theorems of John Bell, Journal of Physics A 47, 424001.

[90] Wolf, S. (2015). Nonlocality without counterfactual reasoning, Physical Review A 92, 052102.

[91] Yanofsky, N.S. (2013). The Outer Limits of Reason (The MIT Press).

[92] Zach, R. (2001). Hilbert's Finitism: Historical, Philosophical, and Metamathematical Perspectives, $\mathrm{PhD}$ Thesis, University of California at Berkeley.

[93] Zvonkin, A.K., Levin, L.A. (1970). The complexity of finite objects and the development of the concepts of information and randomness by means of the theory of algorithms, Russian Mathematical Surveys 25, 83-124. 